\title{
0804 ARE WE USING THE RIGHT DUAL TASK TESTS TO DETECT MOBILITY PROBLEMS? PERSPECTIVES FROM OLDER PEOPLE
}

J Muhaidat*, D A Skelton, A Kerr, C Ballinger, J J Evans Correspondence: School of Health, Glasgow Caledonian University, Govan Mbeki Building, Cowcaddens Road, Glasgow G4 OBA, UK

10.1136/ip.2010.029215.804

Background and Aims Dual tasking is a risk factor for falls. ${ }^{1}$. The aim was to investigate if dual task (DT) laboratory tests use task combinations that community-dwelling older adults perceive as difficult and balance threatening.

Method Two gender-specific focus groups were used. A convenience sample of six males (79.337.45 years) and nine females (74.893.33 years) took part. $80 \%$ fallen at least once. The participants were asked to generate DT examples, rank these in difficulty and then give examples of DT situations that might cause falls. These were then compared to the DT tests used in the literature.

Results There seemed to be a gap between what older people perceive as difficult and the DT tests used to assess performance. Both genders found stairs negotiation accompanied by a manual task (ie, carrying groceries) fall inducing. However, stairs have not been used in the literature to assess DT performance. The Stop Walking When Talking (SWWT) test can classify a person at risk of falling. ${ }^{2}$ The males mentioned that rather than stop walking they adapt and slow down. All participants perceived walking and avoiding moving obstacles (ie, crowds, dogs) as difficult supporting the growing use of functionally relevant DTs. ${ }^{3}$ Conclusion DT tests should be more functionally relevant to older people's activities.

Funding The University of Jordan. Data collection-Fiona Cowan.

\section{References}

1. Swanenburg et al.(2008). BMC Musculoskeletal Disorders, 9:162.

2. Lundin-Olsson et al. (1997) Lancet, 349(9052); 617.

3. Gerin-Lajoie et al.(2006). Gait and Posture, 24: 364-369. 